\title{
Philosophiques
}

\section{Bernard Williams, L'éthique et les limites de la philosophie, traduit de l'anglais par Marie-Anne Lescourret, Paris, Gallimard, 1990.}

\section{Daniel M. Weinstock}

Volume 20, numéro 1, printemps 1993

URI : https://id.erudit.org/iderudit/027222ar

DOI : https://doi.org/10.7202/027222ar

Aller au sommaire du numéro

Éditeur(s)

Société de philosophie du Québec

ISSN

0316-2923 (imprimé)

1492-1391 (numérique)

Découvrir la revue

Citer ce compte rendu

Weinstock, D. M. (1993). Compte rendu de [Bernard Williams, L'éthique et les limites de la philosophie, traduit de l'anglais par Marie-Anne Lescourret, Paris, Gallimard, 1990.] Philosophiques, 20(1), 232-235.

https://doi.org/10.7202/027222ar d'utilisation que vous pouvez consulter en ligne.

https://apropos.erudit.org/fr/usagers/politique-dutilisation/ 


\section{Bernard Williams, L'éthique et les limites de la} philosophie, traduit de l'anglais par Marie-Anne Lescourret, Paris, Gallimard, 1990.

\section{par Daniel M. Weinstock}

Comme son titre l'indique, ce livre du célèbre philosophe britannique Bernard Williams nous invite à remettre en question l'une des principales prétentions de la tradition philosophique occidentale. celle qui consiste à penser que la philosophie serait en mesure de rendre compte de manière théorique et systématique des divers phénomènes qui constituent le champ de l'expérience ethique. Selon Williams, la philosophie, conçue comme un exercice de réflexion et d'articulation à partir des données brutes que fournissent les pratiques éthiques préthéoriques, ou encore le langage moral, ne peut en principe qu'être d'une utilité limitée pour ce qui est « de savoir comment on doit vivre» (p. I).

Disons d'emblée que ce livre constitue une contribution majeure, sainement subversive, au champ de l'ethique philosophique. Depuis sa publication en langue anglaise en 1985, son impact a été considérable; nul ne peut espérer contribuer de manière significative aux débats philosophiques portant sur l'éthique sans s'adresser aux thèses de ce livre. Il est devenu. pour l'instant du moins. incontournable. Il serait vain d'esperer rendre justice en quelques pages à la richesse de l'argumentation que déploie Williams. Je tiens tout simplement dans ce qui suit à en évoquer les moments principaux, et à émettre certaines réserves à l'endroit de ses conclusions.

Dans un premier temps. Williams constate que le type de réponse que les philosophes occidentaux ont, pour la plupart, proposé à la question socratique visant à élucider les grands contours de ce que serait une « vie de bien » (a good life) ne constitue qu'une formulation particulière et limitée. La « moralité »terme à l'aide duquel Williams veut capter le réseau étroit de concepts et de préoccupations des philosophes qui se sont penchés sur la question - ne constitue qu'une partie de l'« éthique », comprise dans son sens le plus large, dénotant « tout schema d'existence susceptible de fournir une réponse intelligible à la question de Socrate » (p. 18). En particulier, les « moralistes » ont selon Williams eu tendance à offrir des analyses « simples » des phénomènes éthiques, en ce sens qu'ils ont cherché à rendre compte de la complexité de la vie éthique à l'aide de théories monistes centrées autour de notions comme « le bien-être », « l'obligation » ou « l'utilité ». Ce réductionnisme serait motivé dans la philosophie morale par le souhait, illusoire selon Williams, qu'ont eu les philosophes de rendre la moralité évaluable selon les termes d'une «conception rationaliste de la rationalité » (p. 24), qui nous fournirait une «monnaie-étalon » en fonction de laquelle nous serions en mesure de juger nos croyances et nos convictions morales. Les philosophes ont également trop eu tendance à isoler certains phénomènes purement « moraux » des autres aspects de nos vies d'agents; selon Williams, une telle délimitation conceptuelle n'est guère possible, puisque ce n'est qu'en tant que membres de communautés et en tant que participants dans leurs pratiques que nous en arrivons à une compréhension de « ce que nous devons faire». 
La philosophie morale a également prétendu que les propositions morales sont susceptibles d'une justification philosophique analogue à celle que nous pouvons trouver pour nos jugements d'observation. Elle a voulu élever la compétence morale au niveau de la connaissance. Selon Williams, cette volonté a souvent été engendrée par l'idée qu'une justification véritablement probante devrait posseder suffisamment de force pour convaincre le sceptique, plutôt tout simplement que ceux qui manifestent déjà le désir de vivre en fonction d'un schème moral. Williams discute longuement les deux tentatives classiques les plus illustres de fournir à la moralité des fondements « indubitables ». celles d'Aristote et de Kant. Ses analyses de ces deux théories sont très différentes : il manifeste une certaine sympathie à l'endroit de la vision morale d'Aristote, car celle-ci met l'accent sur la notion de « vertu » plutôt que sur celle de « règle ». Il estime cependant que la théorie d'Aristote, prise dans son ensemble, doit être rejetée, car la « preuve » qu'elle entend nous fournir du fait que nous ne pouvons pas nous extraire à l'horizon de la moralité dépend de deux thèses que nous ne saurions retenir aujourd'hui : premièrement, la thèse de « l'unité des vertus », selon laquelle il n'est pas possible de posséder une vertu sans les posséder toutes; et une thèse téléologique, ancrée dans la vision biologique aristotélicienne, selon laquelle une saine appréciation de la nature humaine doit nécessairement passer par une appréciation des potentialités humaines et de leur développement « naturel ». Selon Williams, nous pouvons retenir l'accent que porte Aristote sur l'idée que l'agent moral compétent est celui qui possède certaines dispositions affectives le portant à agir correctement plutôt que celui qui aurait à sa disposition un certain nombre de règles appliquées de manière plus ou moins mécanique selon les circonstances, mais l'argumentation dont se sert Aristote afin de fonder la légitimité de cette vision éthique sur une conception de la nature humaine ne nous est plus disponible.

Le fondationalisme de Kant procède selon Williams différemment, mais il échoue tout autant que celui d' Aristote : l'erreur de Kant a consisté en ce que, en voulant nous montrer que la raison pratique elle-même nous impose la reconnaissance de l'impératif catégorique, il nous impose une perspective totalement extérieure à la perspective de la première personne à partir de laquelle nous menons nécessairement nos vies d'agents. Selon Williams, toute conception morale plausible doit pouvoir accommoder le fait que nulle considération qui fait abstraction complète des « projets de base » (ground projects) qui seuls donnent un sens à la vie de l'individu ne peut avoir d'ancrage conatif pour l'être humain. La notion de liberté rationnelle que Kant veut placer aux fondements de sa théorie éthique ne peut avoir d'impact pour la délibération pratique, car celle-ci « s'effectue essentiellement à la première personne, et implique un Je qui doit être lié à mes désirs beaucoup plus intimement que ne l'autorise la conception kantienne » (p. 76 ).

Williams cherche ensuite à remettre en question un nombre important de théories qui, ayant récusé le rêve du fondationalisme, se sont attachées à reconstruire « de l'intérieur » les principes moraux que semble présupposer notre pratique morale. Le contractualisme de Rawls et l'utilitarisme préconisé par des philosophes tels que R.M. Hare sont des exemples importants de cette manière de procéder. Rawls imagine qu'en partant de nos intuitions morales fixes, nous serions en mesure d'élaborer une structure théorique d'un très haut 
niveau de généralité, capable de rendre compte de «nos » diverses intuitions. Hare, pour sa part, élabore un mode de raisonnement faisant appel à la fiction heuristique d'un « observateur idéal » afin de donner une expression théorique au désir que nous avons de maximiser notre bien-être, ainsi qu'a l'engagement envers l'universalisation que notre langage moral nous impose. Hormis un nombre de difficultés techniques, Williams veut également nous faire prendre conscience d'un réflexe philosophique que ces théories incarnent, celui qui consiste à penser que la direction que doit nécessairement adopter la philosophie est celle de l'abstraction, de la réconciliation de points de vue individuels et particuliers par le moyen de structures théoriques très générales. Selon lui, ce réflexe a pour effet de dissimuler la complexité et la richesse de positions éthiques particulières ; plutôt que de nous fournir des outils nous permettant de concilier nos différends, il nous démunit en nous forçant à faire abstraction des concepts moraux « épais 》 (par opposition aux concepts moraux hautement abstraits tels que «juste » et «bien ») auxquels nous n'avons accès qu'en tant que membres de communautés et de pratiques éthiques concrètes. De manière générale, selon Williams, « il est typique de la théorie d'utiliser le présupposé qui veut que nous ayons probablement trop d'idées ethiques, dont certaines peuvent s'avérer de simples préjugés. Or notre probleme essentiel c'est que nous n'en avons pas trop mais au contraire pas assez, et qu'il nous faut en conserver le plus possible » (p. I2g).

La dernière partie du livre, fort intéressante quoique moins bien organisée, cherche à nous suggérer que notre tendance à penser de manière excessivement « théorique » lorsqu'il est question d'éthique témoigne des préjugés scientistes de notre culture. Williams isole un certain nombre d'implications de ce préjugé, dont il estime que nous devrions nous débarrasser : nous avons tendance à penser que nos propositions morales sont composées par un contenu purement descriptif auquel viendraient s'ajouter «de l'extérieur » nos attitudes évaluatives; nous réservons le titre de « connaissance morale » aux pratiques morales dont les principes régulateurs sont susceptibles d'une articulation propositionnelle; nous expliquons de la même manière la convergence éthique et la convergence scientifique, en faisant appel à l'idée d'un monde indeppendant de nos croyances.

La thèse principale du livre, qui se profile en filigrane du début à la fin. est que la philosophie doit cesser de penser qu'elle est en mesure d'ordonner le domaine de l'éthique à l'aide de grands déploiements théoriques, et qu'elle se doit d'adopter une attitude plus humble par rapport à l'éthique, qui consisterait en ce qu'elle se laisse guider davantage par « le vécu éthique », même lorsque celui-ci se refuse à toute systématisation.

Malgré la très grande qualité ainsi que l'importance philosophique de cet ouvrage, je crois qu'il est possible d'émettre un certain nombre de réserves à l'endroit de certaines de ses thèses. En voici deux, très brièvement esquissées : premièrement, l'interprétation que Williams nous offre de Kant n'est en vérité qu'un homme de paille sans intérêt, mais également sans lien réel aux textes de Kant lui-même. Tout d'abord, il ne semble plus plausible, à la lumière des écrits d'Allison, de Rawls et d'O'Neill, de parler de Kant comme d'un fondationaliste. Sa méthodologie, faisant un appel central aux arguments transcendantaux, le placerait plutôt parmi les philosophes qui chercheraient non à 
« prouver » la moralitè, mais à la reconstruire à partir de la conscience morale partagee par des agents moraux compétents. De plus, il me paraît aberrant d'affirmer que Kant veut nous faire adopter à l'endroit de notre vie pratique la même perspective théorique que celle qu'il construit pour rendre compte de l'observation et de la délibération «factuelles ». Cette affirmation repose sur une confusion assez élémentaire qui consiste à identifier la liberté rationnelle à laquelle nous avons accès en tant qu'agents à la liberté qui permet la délibération théorique (p. 75). En effet, Kant estimait que nous ne pouvions guère rendre compte de la possibilite du libre arbitre lorsque nous regardons le monde de manière théorique; c'est justement en nous replaçant dans la perspective de la première personne que nous pouvons conclure que nous sommes (nécessairement) libre. C'est là une stratégie d'argumentation que Williams aurait dû approuver, même en s'éloignant des conclusions substantielles de Kant.

Deuxièmement, Williams commet une erreur caractéristique d'un bon nombre de critiques récents des théories éthiques rationalistes. Il se peut que la compréhension que nous avons de « ce que nous devrions faire » passe souvent par les concepts èthiques « épais » auxquels nous avons accès en tant que membres d'une communauté dont nous partageons la sensibilité, et que cette compréhension soit en principe impossible à articuler en connaissance propositionnelle. C'est là une thèse qui a souvent été répétée depuis quelques années par des philosophes aussi différents que Sandel, MacIntyre et Walzer. Cependant, le monde moderne, celui auquel nos théories éthiques doivent s'appliquer, n'est plus principalement composé par des sociétés homogènes unies par un savoir ethique traditionnel, implicite. Nous devons de plus en plus nous entendre avec d'autres personnes avec lesquelles nous ne partageons rien, si ce n'est la volonté d'en arriver à un accord juste et équitable sur les modalités d'un système de principes pour gouverner nos interactions. Il me semble qu'en vertu de ces conditions, la philosophie, ainsi que sa volonté d'articulation et d'explicitation, ont peut-être un plus grand rôle à jouer dans la détermination de «ce que nous devrions faire » que ne le laisse entendre Williams. Nous ne pouvons nous fier à nos accords intersubjectifs latents si nous voulons réellement construire une éthique pluraliste. La pleine reconnaissance de ce fait chez des philosophes comme Rawls est ce qui me fait penser que leurs theories. malgré leur rationalisme, sont peut-être plus en mesure de nous guider dans nos entreprises morales que ne l'est celle de Williams.

Il faut noter la très grande qualité de la traduction de Marie-Anne Lescourret. Malgré son aspiration à la clarté, la philosophie analytique à laquelle s'identifie Williams (avec quelques réserves importantes évoquées dans sa préface, ainsi que dans un nouvel avant-propos à l'édition française), fait souvent appel à un vocabulaire assez spécialisé ainsi qu'à des tournures de phrase dont le sens n'est souvent pas évident pour le non-initié. Hormis certaines petites maladresses, la traduction du livre de Williams réussit la plupart du temps à éviter les obstacles auxquels doit faire face toute tentative de traduction de textes provenant de la tradition analytique.

Département de philosophie

Columbia University / Université de Montréal 